\title{
HOLOPROSENCEFALIA
}

\section{Análise do seu espectro morfológico em doze casos de autópsia}

\author{
Lúcia de Noronhaㄹ, Ramon Coral Ghanem², Fabíola Medeiros ${ }^{3}$, \\ José Knopfholz², Tiago Augusto Magalhães², Gilberto Antunes Sampaio4, \\ Maria José Serapião ${ }^{5}$, Luiz Fernando Bleggi Torres ${ }^{6}$
}

\begin{abstract}
RESUMO - O termo holoprosencefalia (HPC) é usado para o complexo de deformidades hemisféricas causadas por falha no desenvolvimento da vesícula prosencefálica. Este estudo tem como objetivo relatar o espectro morfológico de 12 casos de HPC, classificá-los e compará-los com a literatura pertinente. Avaliadas 5837 necrópsias realizadas entre 1960 e 1995, 12 apresentavam HPC. Dados como sexo, idade, e presenças de outras malformações associadas foram avaliados e comparados com as demais malformações e com o total de necrópsias. A maioria dos casos era de masculinos $(66,66 \%)$ e neomortos $(75 \%)$. A forma de HPC mais frequentemente encontrada foi a lobar $(58,3 \%)$ e a alteração facial mais frequente foi a ciclopia (25\%). Houve tendência estatística de que a HPC possa, dentro das malformações do sistema nervo central (SNC), acometer mais o sexo masculino e de que a neomortalidade seja maior na HPC do que nas outras malformações do SNC.
\end{abstract}

PALAVRAS-CHAVE: holoprosencefalia, malformações cerebrais, necrópsia.

\section{Holoprosencephaly: morphological aspects of twelve cases of autopsy}

\begin{abstract}
The term holoprosencephaly (HPC) is used to indicate the group of hemispheric deformities caused by a failure in the development of the prosencephalic vesicle. The purpose of this study is to explain the morphologic specter of twelve cases of HPC, qualify them, and compare them to the literature. It was evaluated 5837 pediatrics necropsies, and there were 12 cases of HPC. Data like gender, age and the presence of another associated malformations were evaluated and compared to another malformations and to the total number of necropsies. The majority of the cases was male $(66.66 \%)$, and stillborns (75\%). The most frequently type of HPC found was the lobar type (58.3\%), and the most frequently type of facial alteration was the ciclopy (25\%). There is a statistic tendency to HPC affect the male sex, in comparison with other neurologic malformations. Besides, the stillborns are more frequently observed in HPC than in other neurologic malformations.
\end{abstract}

KEY WORDS: holoprosencephaly, autopsy, nervous system malformations.

O termo holoprosencefalia (HPC) é usado para o complexo de deformidades hemisféricas causadas por falha no desenvolvimento da vesícula prosencefálica. A vesícula prosencefálica éa parte mais cranial do tubo neural. Ela se divide sagitalmente dando origem, bilateralmente, em torno da quinta semana de gestação às vesículas telencefálicas que formarão os dois hemisférios cerebrais. Também se divide transversalmente originando o telencéfalo e diencéfalo, e horizontalmente originando os bulbos olfatórios e vesículas ópticas ${ }^{1}$. Conforme o grau de severidade dessas várias falhas, a HPC pode sofrer as seguintes gradações: alobar, semilobar, lobar e arrinencefalia isolada2,3. Agenesia dos bulbos e dos tractos olfatórios são achados frequentemente associados à holoprosencefalia ${ }^{1,4,5}$, mas podem ocorrer como malformação isolada ou estar presente em outras síndromes ${ }^{1,5}$. Frequentementeencontram-se, também, anor-

Unidade de Patologia Pediátrica e Perinatal (UPPP) do Serviço de Anatomia Patológica (SAP) do Hospital de Clínicas da Universidade Federal do Paraná (HC-UFPR) e Laboratório de Patologia Experimental da Pontifícia Universidade Católica do Paraná (PUC-PR), Curitiba

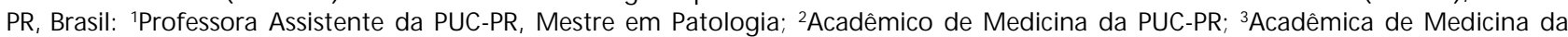
UFPR; ${ }^{4}$ Professor Assistente do Departamento de Patologia Médica da UFPR; ${ }^{5}$ Professora Convidada do Departamento de Patologia Médica da UFPR; ${ }^{6}$ Professor Adjunto da PUC-PR.

Recebido 16 Março 2001, recebido na forma final 1 Agosto 2001. Aceito 6 Agosto 2001.

Dr. Lúcia de Noronha - Centro de Ciências Biológicas e da Saúde - Disciplina de Patologia, PUC-PR - Rua Imaculada Conceição 1155 80215-901 Curitiba PR - Brasil. 
malidades faciais, assim como, ciclopia, cebocefalia, etmocefalia, fenda facial mediana e hipo ou hiperteIorismo ocular-8.

A incidência da HPC é variável, estima-se um valor entre $1 / 16000^{4,9}$ e 1/53000 ${ }^{10}$ nascidos vivos. É 200 vezes mais frequente em crianças de mães diabéticas $^{5,6}$. É geralmente esporádica, entretanto, está frequentementeassociada a aberrações dos cromossomos 13 e 18 etriploidias ${ }^{1,3,4,7}$. Por outro lado, a ocorrência de holoprosencefalia em condições autossômicas recessivas e ligadas ao sexo está bem estabelecida $^{1,3,4,7}$. O prognóstico varia de acordo com o tipo de malformação ${ }^{4,5}$. Em alguns raros casos menos severos, com cérebros bem desenvolvidos, pode-seesperar uma expectativa de vida próxima do normal ${ }^{11}$.

Atualmente, vários estudos têm procurado identificar as alterações genéticas e outros fatores envolvidos na gênese dessa malformação; entretanto, a heterogenicidade etiológica vem dificultando essa investigação. A investigação ultrassonográfica de fetos, realizada sistematicamente ou na presença de oligo/poli-hidrâmnios ou retardo no crescimento intra-uterino podem levar ao diagnostico pré-natal das holoprosencefalias $3,4,12$.

Este estudo tem como objetivo relatar o espectro morfológico de 12 casos de holoprosencefalia, classificá-los e compará-los com a literatura pertinente.

\section{MÉTODO}

Realizaram-se, entre os anos de 1960 e 1995, 5837 necrópsias perinatais e pediátricas, tendo sido verificados 1202 casos (20,59\%) de malformações em geral. As 157 malformações do sistema nervoso central (SNC) representaram $13,06 \%$ das malformações gerais e estiveram presentes em $2,69 \%$ do total de necrópsias estudadas ${ }^{13}$. As necrópsias foram avaliadas e parâmetros como sexo, idade, e presença de outras malformações associadas foram compilados em planilha. A presença de síndrome específica ou simples associação ao acaso foram pesquisadas nos casos selecionados e as HPC foram classificadas conforme os critérios de DeMyer e Zeman²,3 (Tabelas 1 e 2).

Os casos foram subdivididos em grupos etários: natimortos ( $\mathrm{NM}=$ óbito intra-uterino), neomortos ( $\mathrm{NE}=$ óbito até 28 dias), lactentes ( $\mathrm{L}=$ óbito até 11 meses), préescolares ( $\mathrm{PE}=$ óbito até os 6 anos) e escolares $(E=$ óbito até os 14 anos $)^{14}$. A idade ou tempo de vida foi anotada em horas, dias, meses ou anos conforme o grupo etário, sendo que os natimortos apresentam somente idade gestacional dada em semanas já que o seu tempo de vida é igual a zero. As idades gestacionais dos natimortos e neomortos foram calculadas baseadas em dados ecográficos e medidas antropométricas.

Para análise estatística, os dados deste estudo foram divididos em quatro níveis: 1 )total de necrópsias ( $n=5837$ ); 2)total de malformações gerais $(n=1202)$; 3)total de malformações do SNC ( $n=157) ; 4)$ total de casos de HPC $(n=12)$. Utilizou-se o teste do qui-quadrado com nível de significância de $5 \%$ para se verificar se dados como sexo e

Tabela 1. Classificação quanto a forma de apresentação das holoprosencefalias segundo De Meyer.

\begin{tabular}{ll}
\hline Forma & Morfologia cerebral \\
\hline Alobar & Forma mais severa; \\
& Não há divisão entre os hemisférios cerebrais (holosfério); \\
& Cavidade ventricular única (monoventrículo); \\
& Circunvoluções cerebrais aberrantes (paquigiria); \\
& Ausência do corpo caloso e septo pelúcido; \\
& Fusão dos núcleos talâmicos; \\
& Ausência dos bulbos olfatórios. \\
& Forma intermediária; \\
Semilobar & Fissura interhemisférica irregular ou incompleta; \\
& Ponte córticomedular contínua através da linha média; \\
& Monoventrículo freqüentemente dividido em dois cornos temporais posteriores. \\
& Bulbos e tratos olfatórios podem estar bem desenvolvidos. \\
& Forma bem diferenciada; \\
Dois hemisférios bem desenvolvidos; & Ausência do corpo caloso e estruturas da linha média; \\
& Monoventrículo pode salientar-se na região interhemisférica através de uma \\
& formação cística coberta por uma fina camada meningoependimária. \\
Forma menos severa; & Fusência isolada dos bulbos e tractos olfatórios; \\
& Fendas orofaciais podem estar associadas. \\
\hline &
\end{tabular}


Tabela 2. Classificação das anomalias faciais associadas às holoprosencefalias segundo De Meyer e Zemam.

\begin{tabular}{ll}
\hline Grupo & Morfologia facial \\
\hline Anomalia grau I (Ciclopia) & $\begin{array}{l}\text { Fusão das orbitas com presença de apenas um globo ocular ou } \\
\text { dois globos oculares muitos próximos entre si }\end{array}$ \\
Anomalia grau II (Etmocefalia) & $\begin{array}{l}\text { Probócito separa dois olhos hipotelóricos } \\
\text { Ausência do nariz } \\
\text { Microoftalmia }\end{array}$ \\
Anomalia grau III (Cebocefalia) & Hipotelorismo \\
& Nariz achatado, de abertura única \\
& Lábio leporino \\
Anomalia grau IV & Hipotelorismo \\
& Falsa fenda labio-palatina mediana \\
Nariz achatado \\
Hipotelorismo \\
Fenda labial-maxilar bilateral \\
Nariz achatado
\end{tabular}

Tabela 3. Comparação quanto ao sexo entre os casos de HPC, total de necrópsias, total de malformações e total de malformações do SNC.

\begin{tabular}{lcccc}
\hline Sexo & Total de necrópsias & Total de malformações & Malformações do SNC & HPC \\
\hline Masculino & $3129(53,67 \%)$ & $655(54,63 \%)$ & $71(45,51 \%)$ & $8(72,72 \%)$ \\
Feminino & $2701(46,33 \%)$ & $544(45,37 \%)$ & $85(54,49 \%)$ & $3(27,27)$ \\
Total & $5830 *$ & $1199 *$ & $156 *$ & $11^{*}$ \\
\hline
\end{tabular}

* Foram excluídos os casos de intersexo.

mortalidade em determinada faixa etária se mantinham proporcionais ao longo dos níveis citados ou sofriam alguma alteração quando se tratava de HPC.

\section{RESULTADOS}

A prevalência de HPC entre todos os casos de necrópsias pediátricas e perinatais deste estudo (n $=5837$ ) foi $0,21 \%$ e a prevalência de casos de HPC entre todas as malformações ( $n=1202$ ) foi aproximadamente $1,00 \%$. Se considerarmos apenas os casos de malformações do SNC ( $n=157)$ esta prevalência foi $7,64 \%$.

Dentre os 12 casos estudados, $8(66,66 \%)$ eram do sexo masculino, 3 (25\%) do sexo feminino e um caso $(8,33 \%)$ de hermafroditismo verdadeiro (intersexo). Na Tabela 3, nota-se predominância discreta do sexo masculino em três níveis estudados, com predomínio do sexo feminino entre as malformações do SNC. Comparados ambos os sexos no total de necrópsias com o total de malformações em geral encontrou-se $p=0,508$. Quando comparados os 1199 casos de malformações em geral com os 156 casos de malformações do SNC encontrou-se $p=$
0,047. Relacionando-se os casos de malformações do SNC com os casos de HPC obteve-se $p=0,158$. Com relação aos grupos etários, observou-se em todos os grupos estudados uma prevalência de NE. Esta prevalência acentua-se significativamente quando se leva em conta a densidade (número de casos / intervalo de tempo), como é demonstrado na Tabela 4. Quando se verifica a faixa etária dos casos de HPC percebe-se que há maioria de neomortos, não havendo natimortos, pré-escolares e escolares. Dentre os 9 neomortos, 7 eram de termo com tempo de vida médio de 205,85 minutos e 2 eram pré-termo com tempo de vida de 360,5 minutos $(p=0,324)$.

Na análise estatística observou-se uma quantidade menor de óbitos intra-uterinos nas malformações do SNC quando comparadas às malformações em geral ( $p=0,00321)$. Quanto aos casos de HPC comparados com as malformações do SNC em geral houve número nulo de óbitos intra-útero $(p=0,1613)$. Desta forma, há tendência estatística de que a neomortalidade seja maior na HPC do que nas outras malformações do SNC $(p=0,1207)$.

Quanto à forma de apresentação, a mais frequen- 
Tabela 4. Comparação quanto a faixa etária entre os casos de HPC, total de necrópsias, total de malformações e total de malformações do SNC.

\begin{tabular}{ccccccccccccccc}
\hline GE & IT & \multicolumn{3}{c}{ Total de necrópsias } & \multicolumn{2}{c}{ Total de malformações } & \multicolumn{3}{c}{ Malformações do SNC } & \multicolumn{2}{c}{ Total de HPC } \\
& & N & \% & D & N & $\%$ & D & N & $\%$ & D & N & $\%$ & D \\
\hline NM & 280 & 1931 & 33,10 & 6,8964 & 192 & 15,9 & 0,6857 & 54 & 3 & 0,1930 & 0 & 0 & 0,0000 \\
NE & 28 & 2067 & 35,43 & 73,8214 & 534 & 44,4 & 19,0714 & 69 & 47,5 & 2,4643 & 9 & 75 & 0,1071 \\
L & 337 & 940 & 16,1 & 2,7893 & 281 & 23,33 & 0,8338 & 27 & 27,62 & 0,0827 & 3 & 25 & 0,0090 \\
PE & 2190 & 571 & 9,78 & 0,2607 & 127 & 10,54 & 0,0579 & 05 & 11,25 & 0,0010 & 0 & 0 & 0,000 \\
E & 2920 & 328 & 5,61 & 0,1123 & 70 & 5,81 & 0,0239 & 02 & 5,11 & 0,0021 & 0 & 0 & 0,0000 \\
T & 5755 & 5837 & 100 & 1,0142 & 1204 & 100 & 0,0209 & 157 & 100 & 0,0291 & 12 & 100 & 0,0022 \\
\hline
\end{tabular}

GE, grupo etário; IT, tempo de duração do grupo etário específico; NM, natimorto; NE, neomorto; L, lactente; PE, préescolar; E, escolar; T, total; N, número de casos; \%, porcentagem do grupo etário em relação ao total; D, densidade (N/TT).

Tabela 5. Malformações associadas às HPC (os três pacientes com trissomias foram excluídos) $(n=9)$.

\begin{tabular}{ll}
\hline & № casos (\%) \\
\hline Malformações renais & \\
Hipoplasia adrenal & $4(44,4 \%)$ \\
Displasia renal cística & $2(22,2 \%)$ \\
Hipoplasia renal & $1(11,1 \%)$ \\
Hiperplasia adrenal & $1(11,1 \%)$ \\
Sem alterações & $1(11,1 \%)$ \\
Outras malformações & \\
Cia & $1(11,1 \%)$ \\
Trilogia de Fallot/ pulmonar bicúspide & $1(11,1 \%)$ \\
Prega palmar única & $2(22,2 \%)$ \\
Polidactilia & $1(11,1 \%)$ \\
Micropênis & $2(22,2 \%)$ \\
Hipoplasia testicular & $4(44,4 \%)$ \\
Hipoplasia de pulmão & $1(11,1 \%)$ \\
Pulmão esquerdo trilobado & $1(11,1 \%)$ \\
Divertículo de Meckel & $1(11,1 \%)$ \\
\hline
\end{tabular}

te foi a forma lobar, presente em $58,3 \%(n=7)$ dos casos. As formas semilobar ealobar estiveram ambas presentes em $16,7 \%(n=2)$ dos casos. Houve um caso de arrinencefalia isolada (8,3\%).

As alterações faciais estão demonstradas na Figura 1. Quanto às malformações associadas observamos que $25 \%$ dos casos apresentaram anormalidades que em conjunto representavam uma síndrome específica ou cromossomopatia, a HPC fazendo parte do espectro deste fenótipo. Havia dois casos de trissomia do $13(16,7 \%)$ e um caso trissomia do 18 (8,3\%). Outras malformações congênitas de diversos órgãos ou sistemas foram encontradas associadas com as HPC porém sem caracterizar um fenótipo sindrômico, parecendo se tratar de puro acaso ou formas frustras de alterações cromossomiais. Estas malformações são demonstradas na Tabela 5.

As Figuras 2, 3 e 4 ilustram nossos achados.

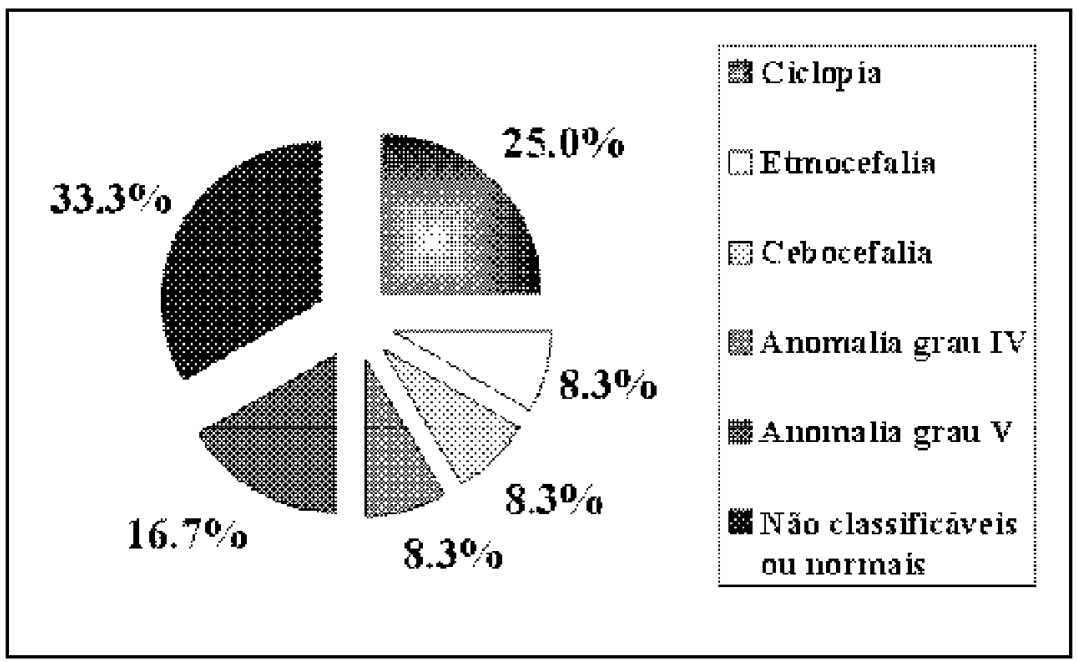

Fig 1. Alterações faciais encontradas. 


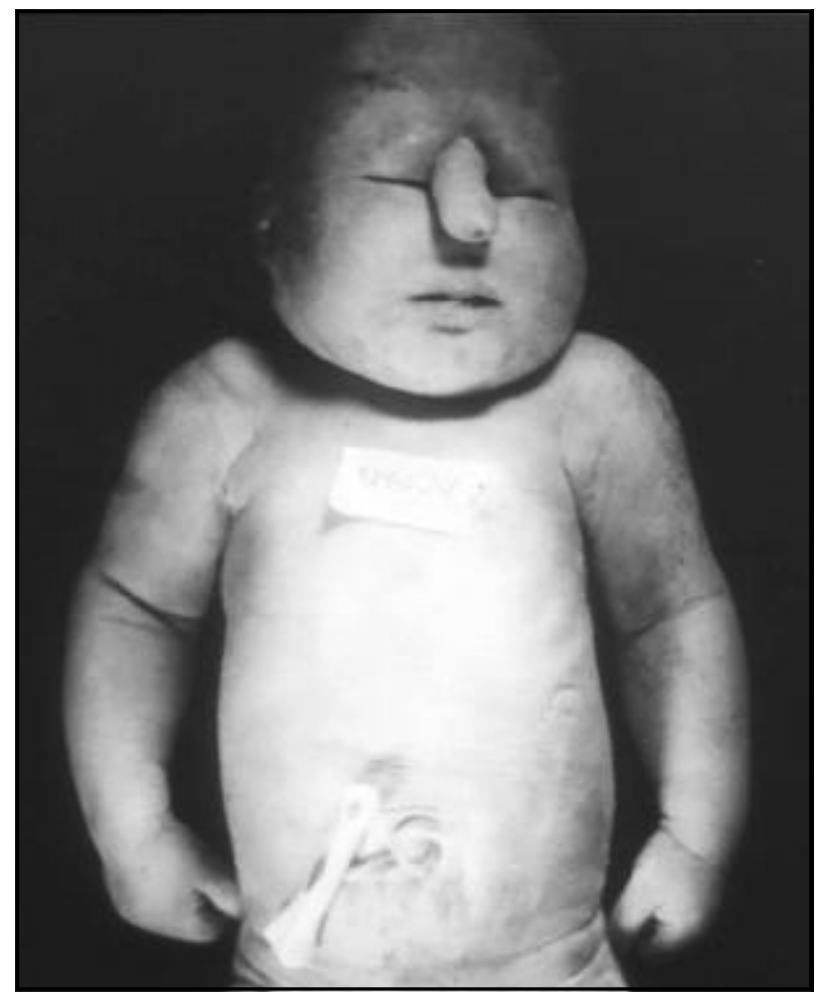

Fig 2. Face de um dos casos deste estudo com alterações características de etmocefalia. Presença de probócide associada a hipertelorismo, ausência de nariz e microftalmia.

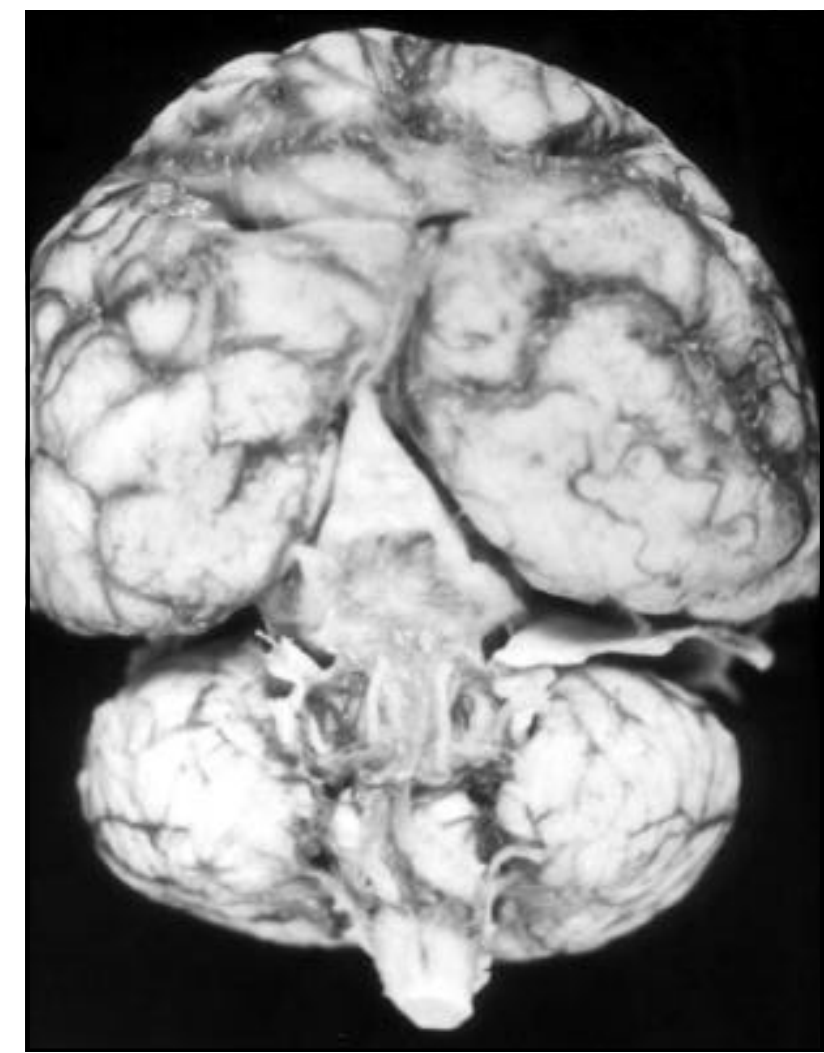

Fig 3. Face posterior do encéfalo em que se observa fusão quase completa dos hemisférios cerebrais. Apenas o lobo occiptal apresenta-se parcialmente dividido na linha média. A fissura interhemisférica é então irregular e incompleta caracterizando a forma semilobar.
Fig 4. Cortes coronais de um caso de forma lobar de holoprosencefalia. Esta é uma forma bem diferenciada em que os dois hemisférios estão bem desenvolvidos, porém observa-se ausência do corpo caloso e um monoventrículo.

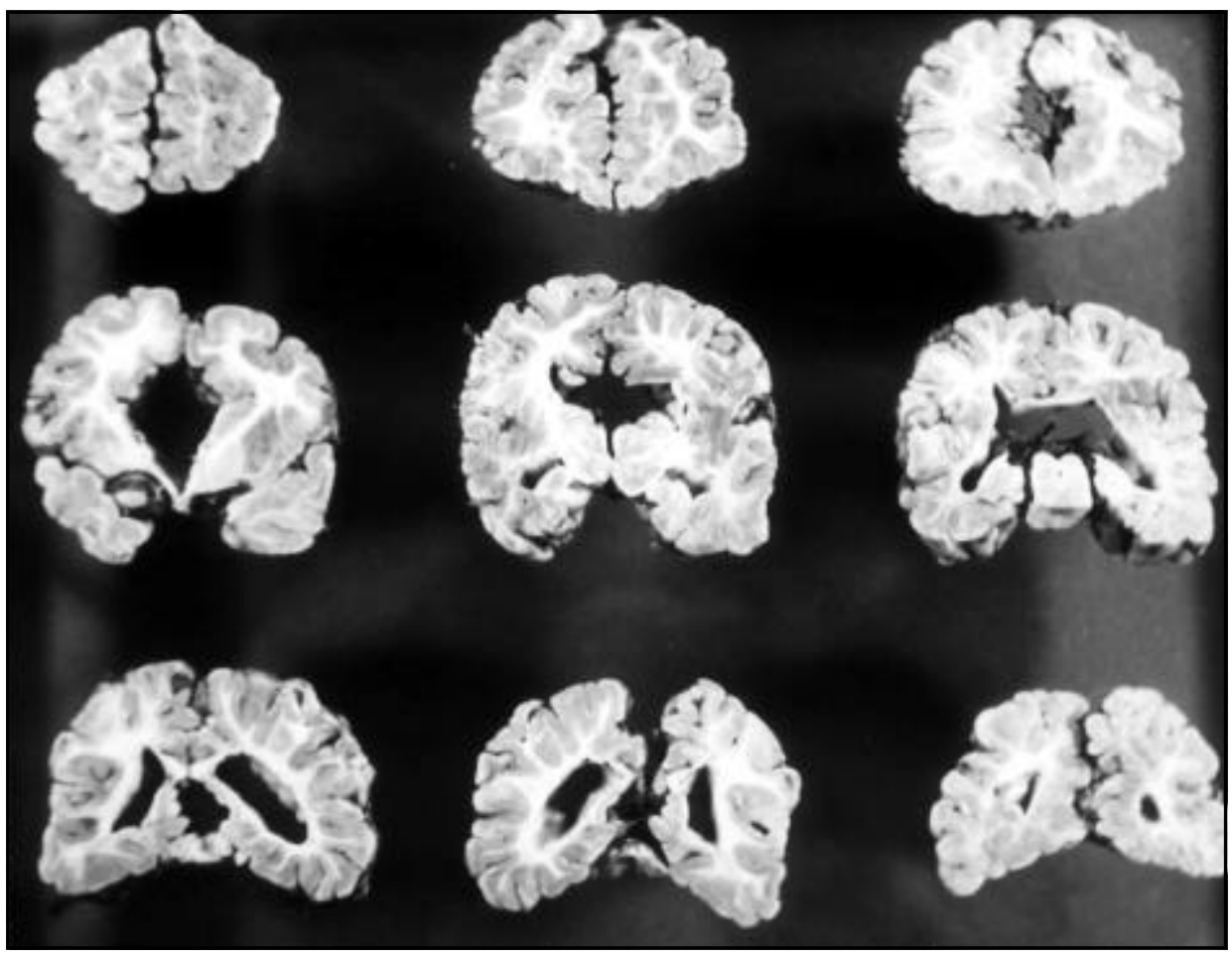




\section{DISCUSSÃO}

A prevalência de HPC foi $7,64 \%$ dentre as malformações do SNC, o que representou $1,00 \%$ de todos os malformados e $0,21 \%$ de todas as necrópsias perinatais e pediátricas realizadas no HC/UFPR entre os anos de 1960 e 1995. Matsunaga et al. (citados por Chervenak et al. ${ }^{4}$ ) encontraram, em 1977, em estudo epidemiológico de 150 embriões com HPC, incidência de 1:250 abortos espontâneos $(0,4 \%)$. Dentre nascidos vivos calcula-se uma incidência entre $1 / 16000^{4,9}$ e $1 / 53000^{10}(0,006$ e $0,002 \%)$.

Pelo estudo estatístico, concluiu-se que dentre as 5830 necrópsias estudadas o fato de ter ou não uma malformação independe do sexo da criança, ou seja, os casos de malformação se distribuem de forma proporcional segundo o sexo $(p=0,5058)$. Assim como existem mais crianças do sexo masculino no todo, isto também ocorre entre os malformados. Entretanto, se compararmos os 1199 casos de malformações gerais com os casos de malformações do SNC $(n=157)$, observamos neste último maior prevalência do sexo feminino, havendo diferença estatisticamente significativa $(p=0,047)$. Este valor de " $p$ " indica uma tendência das malformações do SNC ocorrerem no sexo feminino. A explicação para este fato se dá quando se verifica que defeito do tubo neural, malformação do SNC mais frequente, é mais prevalente no sexo feminino ${ }^{13}$. Em nossa amostra a predominância foi do sexo masculino $(2,67: 1)$. Isso sugere uma tendência estatística de que a HPC possa, dentro das malformações do SNC, acometer mais o sexo masculino. Observou-se, entretanto, que os casos de malformação do SNC quando comparados aos de HPC não demonstram diferença estatística quanto ao sexo da criança $(p=$ $0,157)$, talvez pelo reduzido número de casos de HPC encontrados nessa amostra. Roach et al. ${ }^{9}$ encontraram uma predominância do sexo feminino na proporção de 3:1, praticamente uma inversão de valores. Alguns autores, entretanto, acreditam que não há preferência entre os sexos ${ }^{1}$, enquanto outros defendem que a incidência varia, não podendo ser bem determinada ${ }^{15}$.

O tempo de vida foi inversamente proporcional à severidade das malformações. Assim, os NE com a forma alobar e semilobar tiveram tempo de vida médio de 19,5 horas. Já os NE e L que possuíam a forma lobar, de pequena gravidade, viveram em média 76,5 dias, variando de 30 minutos a 11 meses. Os neomortos a termo tiveram estatisticamente o mesmo tempo de vida que os pré-termo, não parecendo interferir a idade gestacional na sobrevida dos portadores de HPC, desde que compatível com a vida.

A observação da Tabela 4, associada ao estudo estatístico, demonstra que as malformações do SNC levam menos a óbito intra-uterino que as malformações em geral. Por isto, ocorreram mais casos de neomortos elactentes entre os casos com malformações do SNC. As tendências estatísticas levam a crer que, se houvesse amostra maior, seria possível constatar quea HPC modificaria o período em que o óbito vai ocorrer, quando comparado às demais malformações do SNC. Isto sugere que a HPC tende a determinar mais o óbito no período pós-natal imediato que as outras malformações neurológicas.

A forma de HPC mais frequentemente encontrada foi a lobar (58,3\%), seguida pela forma alobar $(16,7 \%)$, semilobar $(16,7 \%)$ e arrinencefalia isolada (8,3\%). Parant et al. encontraram dentre 12 casos de HPC, 11 (91\%) da forma alobar e apenas um semilobar $^{12}$. Em outro estudo ${ }^{5}$, dentre 6 casos de HPC, foram encontrados dois alobares, três semilobares e um lobar. Pode-se perceber uma grande variabilidade nos resultados, o que se credita às pequenas amostras relatadas na literatura devido à raridade desta malformação ou a eventuais associações com outras sindromes.

A ciclopia, alteração facial mais severa, foi a mais frequente, seguida pela anomalia grau $\mathrm{V}$. A etmocefalia, a anormalidade facial menos comum foi vista em apenas um caso (8,3\%).

Um caso apresentava implantação baixa das oreIhas e microoftalmia que, não podendo ser classificado, foi considerado um caso transicional entre as formas menos severas de alterações faciais.

Segundo DeMyer et al. ${ }^{8}$, as alterações faciais e craniais encontradas nos casos de holoprosencefalia são geralmente proporcionais àquelas encontradas no cérebro. Assim, quanto mais graves as alterações faciais presentes, maior a probabilidade de que a HPC seja alobar, com baixas perspectivas de vida, sendo importante indicador prognóstico. Entretanto, neste estudo, esta correlação só foi verdadeira em $75 \%$ dos casos, o que coincide com outro estudo onde encontrou-se $80 \%$ de correlação ${ }^{11}$.

Cerca de $25 \%$ dos casos apresentaram cromossomopatias, sendo dois casos de trissomia do 13 $(16,7 \%)$ e um caso trissomia do 18 (8,3\%). Aproximadamente metade dos pacientes não apresenta alterações cromossômicas, entretanto éfrequente a presença de trissomia do 13 em pacientes com HPC associada a múltiplas malformações viscerais ${ }^{1}$. Asanor- 
malidades cromossômicas estão frequentemente ausentes quando a HPC é a única malformação ${ }^{8}$, mas na presença de comprometimento multissistêmico, estas são frequentes, embora haja exceções ${ }^{5,8}$.

Quanto a outras malformações não relacionadas a cromossomopatias, as alterações renais e adrenais foram muito frequentes nessa amostra. As mais frequentes foram a hipoplasia adrenal, presente em $44,4 \%$ dos casos e a displasia renal cística, presente em $22,2 \%$ dos casos. Muitos autores relataram a presença de hipoplasia adrenal e tireoideana nesses pacientes ${ }^{1,5}$. Existem registros na literatura sobre associação de HPC com anoftalmia, pés tortos, hidrocefalia, defeitos diafragmáticos, onfalocele, malformações de fígado, vagina, hipófise, coração, além de outras ${ }^{5,6,15}$. Neste estudo, as formas mais graves de HPC estiveram acompanhadas de outras malformações.

A riqueza de variações associadas à falta de informações sobre o assunto tornam a HPC diagnóstico muitas vezes pouco cogitado, embora esta doença seja relativamente prevalente dentro das malformações de SNC.

\section{REFERÊNCIAS}

1. Friede RL. Developmental neuropathology. 2.Ed. N ew York: SpringerVerlag, 1989:296-308.

2. DeMeyer W. Classification of cerebral malformations. Birth Defects 1971;7:78-93.

3. Duckett S. Pediatric neuropathology. Philadelphia: Lippincott Williams \& Wilkins, 1995:108-122.

4. Chervenak FA, Isaacson G, Mahoney MJ, et al. Theobstetric significance of holoprosencephaly. Obstet Gynecol 1984;63:115-121.

5. Lamego IS, Barbosa-Coutinho LM. Holoprosencefalia: estudo de seis casos. Arq Neuropsiquiatr 1994;52:523-529.

6. Barr, M Jr, Hanson JW, Currey K, et al. Holoprosencephaly in infants of diabetic mothers. J Pediatr 1983;102:565-568.

7. Diament A, Cypel S. Neurologia infantil, capítulo 13. 2.Ed. São Paulo: Atheneu, 1989:112-135.

8. DeM eyer WE, Zeman W. Familial alobar holoprosencephaly (arhinencephaly) with median cleft lip and palate: clinical, encephalografic and nosologic considerations. Neurology 1963;13:913-918.

9. Roach E, DeM eyer WE, Palmer K, et al. Holoprosencephaly: birth data, genetic and demographic analysis of 30 families. Birth Defects 1975;11:294-313.

10. Saunders ES, Shortland D, Dunn PM. What is the incidence of holoprosencephaly? J Med Genet 1984;21:21-26.

11. Elias DL, Kawamoto HK Jr, Wilson LF. Holoprosencephaly and midline facial anomalies: redefining classification and management. Plast Reconstr Surg 1992;90:951-958.

12. Parant $O$, Sarramon MF, Delisle MB, Fournié A. Prenatal diagnosis of holoprosencephaly: a series of twelve cases. J Gynecol Obstet Biol Reprod 1997;26:687-696.

13. Noronha L, Medeiros F, Martins VDM, et al. Malformações do SNC: análise de 157 necrópsias pediátricas. Arq Neuropsiquiatr 2000;58:890-896.

14. Nelson WE. Tratado de Pediatria. 15.Ed. Rio de Janeiro: GuanabaraKoogan, 1997:35-77.

15. Stevenson DK, Sunshine P. Fetal \& neonatal brain injury: mechanisms, management and therisks of practice, chapter 11. 2.Ed. Oxford: Oxford Univ Press, 1997:107-123. 